\title{
200 Jahre Ärztegesellschaft des Kantons Bern
}
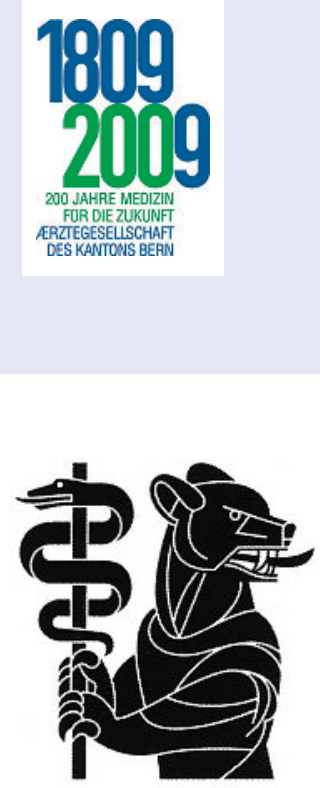

ÆRZTEGESELLSCHAFT DES KANTONS BERN SOCIETE DES MEDECINS DU CANTON DE BERNE

Urs Boschung

Direktor des Instituts für Medizingeschichte, Universität Bern

\author{
Die 1809 in Burgdorf gegründete Ärztegesellschaft des Kantons Bern ist die älteste \\ ununterbrochen tätige kantonale Ärztevereinigung der Schweiz. Von den Anfän- \\ gen bis heute verfolgt sie das Ziel, die Ärzteschaft zu vereinigen, zu Fragen des \\ Gesundheitswesens und der Standespolitik Stellung zu nehmen und zur prakti- \\ schen Umsetzung wissenschaftlicher Erkenntnisse beizutragen.
}

\section{9: Die Gründung}

Am 9. Oktober 1809 trafen sich in Burgdorf 21 Ärzte zur Gründung der «Medicinisch-chirurgischen Gesellschaft des Cantons Bern». In zwei früheren Versammlungen hatte man sich bereits über die Hauptziele «Pflege von Collegialität und Wissenschaft» geeinigt. Von den 46 bis Ende 1809 beigetretenen Mitgliedern waren 13 Doktor der Medizin, 31 trugen den Titel Arzt und/oder Wundarzt, 2 waren Apotheker. 10 Mitglieder (davon 5 Dr. med.) wohnten in Bern, 5 in Thun und Burgdorf, 31 auf dem Land. Es galt nun, die akademischen Ärzte und die handwerklich ausgebildeten Wundärzte und Chirurgen und damit auch Stadt und Land einander näherzubringen, was durch die veränderten politischen Verhältnisse wesentlich erleichtert wurde. Bezeichnend für den sich anbahnenden Wandel ist die Laufbahn der beiden ersten Präsidenten. Der «Gründer» Samuel Lehmann (1769-1837) von Langnau verfügte über ein kantonales Patent als «Arzt und Wundarzt». Er hatte nach der Wundarztlehre und Gesellenzeit sein Wissen am Zürcher Medizinischchirurgischen Institut vertieft und 1789 in Bern vor der (handwerklichen) «Chirurgischen Societät» sein Meisterexamen bestanden. Rudolf Abraham Schiferli
(1775-1837), Präsident 1809-1812, war Bürger von Thun, Dr. med. der Universität Jena und Professor der Chirurgie an der Berner Akademie, die in einem vierjährigen Curriculum Landärzte ausbildete und die Anspruchsvolleren auf das Studium und Doktorat an einer auswärtigen Universität vorbereitete.

\section{Jahrhundert: Wirken in Kanton und Bund} In den ersten hundert Jahren wuchs die Gesellschaft zu «kräftigem, achtunggebietendem Mannesalter» heran. Um 1900 zählte sie rund 300 Mitglieder, darunter drei Frauen. Viele von ihnen hatten an der Berner Medizinischen Fakultät studiert, die mit Theodor Kocher, Theodor Langhans und Hermann Sahli in hohem Ansehen stand und über ein neues Spital und grosszügige Institutsbauten verfügte. Durch das Freizügigkeitsgesetz von 1877 und die Eidg. Medizinalprüfungen war das Curriculum klar geregelt. Das Medizinstudium der Frauen setzte sich durch, zuerst vor allem dank dem Zuzug der Russinnen, denen zögernd auch die Schweizerinnen folgten. Eine spezielle Weiterbildung war namentlich in den operativen Fächern bereits zur Selbstverständlichkeit geworden. Die Spitäler, die seit der Gründung der «Notfallstuben» (1835) im
Sommersitzung der Medizinisch-chirurgischen Gesellschaft des Kantons Bern auf der Petersinsel, 16. Juli 1887, Präsident Theodor Kocher mit dem Isenschmid-Becher. Beschriftung von Kochers Hand. (Institut für Medizingeschichte, Universität Bern)
Korrespondenz:

Prof. Dr. med. Urs Boschung Institut für Medizingeschichte Bühlstrasse 26

Postfach

CH-3000 Bern 9

urs.boschung@mhi.unibe.ch

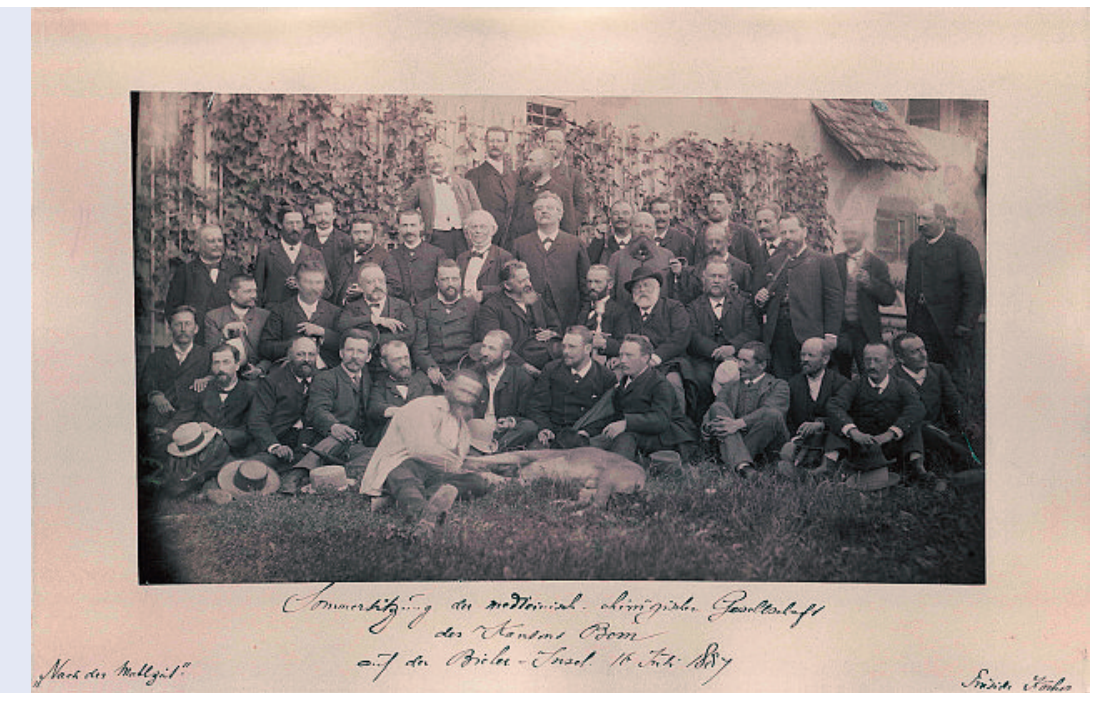


ganzen Kanton laufend ausgebaut wurden, boten entsprechende Assistenz- und Chefarztstellen. Die in den 1840er Jahren als regionale Zusammenschlüsse entstandenen Bezirksvereine stellten die alltagsnahe ärztliche Fortbildung sicher, während die Kantonalgesellschaft zu Fragen der Volksgesundheit und der Spitalplanung Stellung bezog und sich für eine vermehrte Zusammenarbeit der Ärztevereinigungen auf Bundesebene einsetzte.

Dank einer Berner Initiative gründeten 1870 im Eisenbahnknotenpunkt Olten Delegierte der deutschschweizerischen Gesellschaften den Ärztlichen Centralverein der deutschen Schweiz mit dem Zweck «wissenschaftlicher und social-medizinischer Besprechungen». Erster Vorsitzender war der Berner Kantonalpräsident Dr. Johann Rudolf Schneider (1804-1880). Noch im gleichen Jahr wurde die Herausgabe eines «schweizerischen Journals, das die Interessen der Ärzte in ziviler und militärischer Beziehung vertrete», beschlossen und die Redaktion dem Berner Professor Edwin Klebs übertragen. So erschien von 1871 an das CorrespondenzBlatt für Schweizer Aerzte, das wichtigste Organ für medizinische Wissenschaft und Standesfragen bis zu seiner Aufteilung in Schweizerische Ärztezeitung und Schweizerische Medizinische Wochenschrift 1920.

Der nächste Schritt wurde 1873 getan, als auf Initiative von J. R. Schneider sich 160 Mitglieder des Centralvereins und der Société médicale de la Suisse romande (gegründet 1867) in Bern versammelten. Alle zwei Jahre traf man sich in der Folge wieder in Bern. 1875 wurde die Bildung der gesamtschweizerischen, von den Bundesbehörden als Expertengremium anerkannten Ärztekommission bekanntgegeben. Am Ersten schweize-

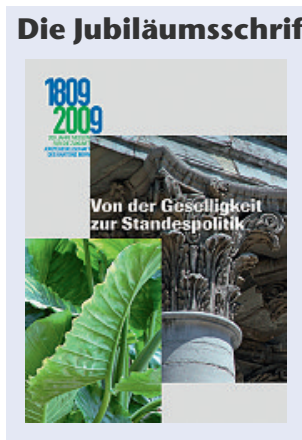

Von der Geselligkeit zur Stan despolitik. 200 Jahre Ärztegesellschaft des Kantons Bern. Bern 2008, 234 S., ill., CDROM. Im Auftrag des Vorstandes herausgegeben von einer Arbeitsgruppe (Urs Boschung, Madeleine Herren, Jürg Schlup, Werner Ringli, Reto Steiner, Roland Brechbühler, Daniel Lüthi, Piroschka Wolf). Redaktion: Urs Boschung

Mit Beiträgen von Jürg Schlup, Urs Boschung, Madeleine Herren, Eberhard Wolff, Martin Lengwiler, Franziska Rogger, Anouk Hiedl, Simona Isler. - Statements von Christine Aebi-Ochsner, Esther Fischer-Homberger, Max Geiser, Benedikt Horn, Marco Mumenthaler, André Piguet, Werner Ringli, Hans Rudolf Sahli, Jürg Steiger.

Die Jubiläumsschrift legt den Schwerpunkt ins 20. Jahrhundert, in dessen Verlauf sich die ärztliche rischen Ärztetag, an dem nun auch die Tessiner Kollegen teilnahmen, begrüsste Theodor Kocher am 30. Mai 1885 in Bern die zahlreich erschienenen «ünger Aesculaps» im Hörsaal der Chirurgischen Klinik des neuen Inselspitals. Im Turnus mit Basel und Zürich fanden von da an Schweizerische Ärztetage mit jeweils rund 300 Teilnehmern statt. An diesen Anlässen reifte die Idee von der Notwendigkeit einer repräsentativen Ärzteorganisation auf Bundesebene. 1901 trafen sich Delegierte aller schweizerischen Ärztegesellschaften in Olten zu Beratungen über die Gründung der Verbindung der schweizerischen Aerzte FMH, bestehend aus der Ärztekammer, d.h. Abgeordneten der Kantonalgesellschaften proportional der Mitgliederzahl, und der Ärztekommission als Exekutivorgan. 1902 trat die FMH auf den Plan, während in ihrem jeweiligen Einzugsbereich der Zentralverein und die Société médicale de la Suisse romande weiterhin tätig blieben.

\section{Jahrhundert: Vorrang der Standespolitik}

Gerade zum Zeitpunkt des 100-Jahr-Jubiläums herrschte in der Ärzteschaft der Eindruck einer schweren Krise, die Umdenken und entschiedenes Handeln erforderte. Sinkende Geltung des praktizierenden Arztes, verschlimmerte ökonomische Lage, Verstaatlichungsideen «in den sozialistischen Kreisen», Konkurrenzkampf unter den Ärzten und mit «medizinischen Sekten», dies alles erforderte - wie 1907 Dr. Friedrich Ris aus Thun darlegte - die «Organisierung der Ärzte zu einem Stande, was sie bisher nicht gewesen, zu Schutz und Trutz gegen das Publikum, gegen die andern Stände». In der Aufzählung nicht namentlich genannt, jedoch am wichtigsten war die Tatsache,

Tätigkeit und ihr Umfeld grundlegend wandelten und die Stellung des Arztes und der Ärztin im Gesundheitswesen durch Politik, Krankenkassen und weitere gesellschaftliche Faktoren zunehmend beeinflusst wurde. Die Fachbeiträge werden ergänzt durch persönliche Statements, in denen Ärztinnen und Ärzte zu Grundsatzfragen Stellung nehmen und Rückschau, Umschau und Ausschau halten. Die beigelegte DVD enthält Illustrationen und digitalisierte Dokumente, u. a. die Festschrift und das Liederbuch von 1909 (mit Tonaufnahmen der Berner Singstudenten) und eine Porträtgalerie von Berner Ärztinnen und Ärzten, fotografiert von Kollege Peter Friedli.

Die Jubiläumsschrift kann beim Sekretariat der Ärztegesellschaft des Kantons Bern zum Selbstkostenpreis von Fr. 31.-, zuzüglich Versandspesen von Fr. 8.-, bezogen werden. Mitglieder der Ärztegesellschaft erhalten ein Exemplar unentgeltlich.

www.berner-aerzte.ch/kontakt.php 
dass in zunehmendem Mass die Krankenkassen das Arzt-Patienten-Verhältnis mitbestimmten und dass eine gesetzliche Regelung der Kranken- und Unfallversicherung auf nationaler Ebene bevorstand. Ein doppeltes Obligatorium wurde zwar 1900 vom Stimmvolk verworfen, jedoch 1912 für die Unfallversicherung angenommen.

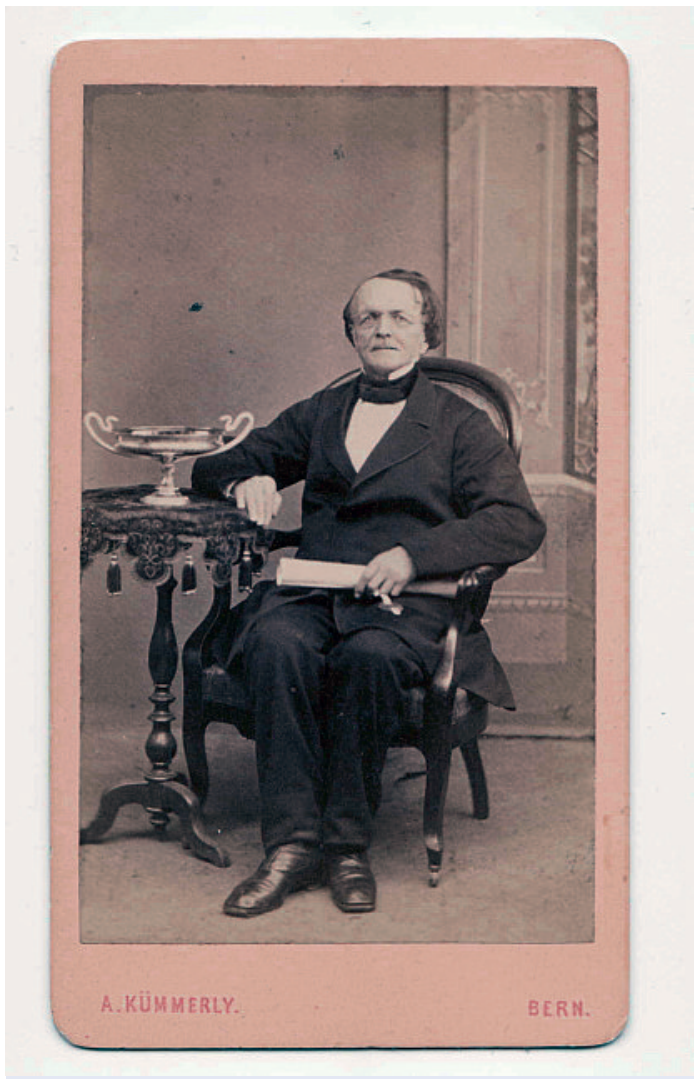

Dr. med. Johann Rudolf Schneider (1804-1880),

Präsident 1859-1880, mit der Schale, die ihm die Berner Ärztegesellschaft 1870 zum Dank für die geleisteten Dienste überreichte. (Institut für Medizingeschichte, Universität Bern)
Um inskünftig in Sachen «Kassenmedizin» geschlossen auftreten zu können, gab sich die Gesellschaft in der Urabstimmung von 1911 den neuen Namen Aerztegesellschaft des Kantons Bern und revidierte Statuten mit einer verbindlichen Standesordnung, wie dies der Zentralverein 1904 empfohlen hatte. Künftig war es nicht mehr gestattet, dass wie bisher einzelne Mitglieder mit Krankenkassen Sondertarife aushandelten. Dafür war nun allein die Kantonalgesellschaft zuständig. Standespolitik wurde damit zur wichtigsten Aufgabe der Ärztegesellschaft. Ab 1914 galt erstmals ein von der Berner Ärztegesellschaft mit den Kassen ausgehandelter, vom Regierungsrat bestätigter Tarif. Diskussionsstoff gab das kantonale Krankenversicherungsobligatorium und dessen Ausdehnung von den ärmeren Schichten auf den Mittelstand und auf besser situierte Kreise. 1933 bewilligte die Berner Ärzteschaft im Gegensatz zu den Zürcher Kollegen einen Krisenrabatt von $10 \%$, was das Verhältnis zu den Kassen entspannte, so dass in der Folge Tarifanpassungen in einer «paritätischen Kommission» gemeinsam erarbeitet werden konnten.

Mit der zunehmenden Spezialisierung verschärfte sich nach dem 2. Weltkrieg der Gegensatz zwischen den Land- und Stadtärzten. In den 1960er Jahren wurde die «Kostenexplosion im Gesundheitswesen» zum Dauerthema. Um 1980 stellte die Ärztegesellschaft aufgrund eigener Erhebungen fest, dass in den Praxen die Einkommen faktisch stagnierten, dass die Kostenzunahme vielmehr aus der steigenden Ärztedichte resultierte. In der Zeit von 1960 bis 1985 verdoppelte sich die Zahl der Mitglieder der Gesellschaft von 800 auf 1600, während die Zahl der Einwohner des Kantons im gleichen Zeitraum nur um etwa ein Prozent zunahm. Das schon bei der Gründung bestehende Gefälle zwischen Zentrum und Peripherie hatte sich verstärkt. 1990 wohnten 50\% aller Ärzte des Kantons in der Gemeinde Bern und nahezu 75\% in Gemeinden mit mehr als 10000 Einwohnern (gem. Historischstatistischer Atlas des Kantons Bern, 1989, S. 134). 